Data Repository for: "Climatic and tectonic controls on sedimentation and erosion during the Pliocene-Quaternary in Qaidam Basin (China),” by Richard V. Heermance, Alex Pullen, Paul Kapp, Carmala N. Garzione, Scott Bogue, Lin Ding, and Peiping Song; doi: 10.1130/B30748.1

\title{
Magnetostratigraphy Analysis
}

Our section sampled the Plio-Quaternary part of the stratigraphic section, and previous work on similar strata within the Qaidam Basin documented sediment accumulation rates of 200-900 m/m.y (Liu et al., 1998; Fang et al., 2008; Zhang et al., 2012a). Assuming that PlioQuaternary Qaidam strata accumulated at approximately same rate, each $17 \mathrm{~m}$-interval spans between $0.018-0.085$ million years (m.y.). The average duration of a polarity chron since the start of the Pliocene (the suspected maximum age of our strata) is $0.251 \mathrm{~m}$.y. (calculated from Lourens et al., 2004), and so we anticipated that each polarity interval should be defined by three paleomagnetic sites on average. Excluding the two polarity intervals that span less than 20,000 years (Cobb Mountain and Reunion Subchrons at 1.1 and 2.1 Ma), the shortest polarity chron in the interval lasted $0.084 \mathrm{~m} . \mathrm{y}$. and so should be represented by a least one paleomagnetic site.

Samples were analyzed at the paleomagnetic laboratory at Occidental College. Remanent magnetization was measured using a 3-axis DC-SQUID magnetometer system housed in a magnetically shielded room. The magnetometer has a background noise of $<1$ picoAm ${ }^{2}$ and is equipped with a vacuum pick-and-put, computer-controlled sample handling system which can measure up to 180 samples automatically (Kirschvink et al. 2008). Alternating field (AF) demagnetization was performed with a computer-controlled, two-axis coil system. Thermal demagnetization was performed in a commercially built, magnetically shielded furnace.

\section{Paleomagnetic Sample Analysis}

One sample per site or stratigraphic level was initially measured for natural remanent magnetization (NRM), then subjected to AF demagnetization in four steps up to $20 \mathrm{mT}$ to remove low-coercivity magnetizations, and subsequently treated with stepwise thermal demagnetization using 6-12 demagnetization steps between $200-680{ }^{\circ} \mathrm{C}$, typically in $50{ }^{\circ} \mathrm{C}$ steps up to $500^{\circ} \mathrm{C}$ and $30^{\circ}$ steps from $500{ }^{\circ} \mathrm{C}$ to $680^{\circ} \mathrm{C}$.

Some samples, particularly from greenish-gray gypsiferous lacustrine beds near the top of the section, had very low NRM values $\left(<10^{-4} \mathrm{~A} / \mathrm{m}\right)$. For these samples, the typical thermal demagnetization sequence to at least $580^{\circ}$ yielded erratic, uninterpretable results (Fig. $7 \mathrm{E}$ ). Instead, these samples were submitted to three steps of AF demagnetization (3, 6, and $9 \mathrm{mT})$ and then small, $10-50{ }^{\circ} \mathrm{C}$ steps between $140^{\circ}$ and $350^{\circ}$, after which the typical thermal demagnetization sequence was continued. This method was intended to isolate the magnetic remanence from iron-suphides that have lower unblocking temperatures but can carry primary magnetic remanence in some low-energy depositional environments (e.g., Husing et al., 2007).

To determine characteristic magnetic remanence (ChRM) directions, line-fit analysis (Kirschvink, 1980) was performed for each specimen based on data from a minimum of four but typically seven or eight thermal demagnetization steps using paleomagnetic software of Jones (2002). Samples were accepted as usable when the maximum angular deviation (MAD) of the line fit was less than $15^{\circ}$. In cases where all but the final HTC point clustered in a normal or reverse polarity position on an orthogonal vector plot, the ChRM direction was determined by forcing a line from the cluster through the origin. At each site, a second and third specimen from the block-sample was demagnetized if the first yielded unstable or overprinted directions or if a magnetostratigraphic interval of normal or reverse polarity was defined by only one specimen. 


\section{REFERENCES CITED}

Fang, X., Zhang, W., Meng, Q., Gao, J., Wang, X., King, J., Song, C., Dai, S., and Miao, Y., 2007, High-resolution magnetostratigraphy of the Neogene Huaitoutala section in the eastern Qaidam Basin on the NE Tibetan Plateau, Qinghai Province, China, and its implication on tectonic uplift of the NE Tibetan Plateau: Earth and Planetary Science Letters, v. 258, no. 1, p. 293-306, doi:10.1016/j.epsl.2007.03.042.

Fang, X., Wu, F., Han, W., Wang, Y., Zhang, X., and Zhang, W., 2008, Plio-Pleistocene drying process of Asian inland-Sporopollen and salinity records from Yahu section in the central Qaidam Basin: Quaternary Sciences, v. 28, no. 5, p. 875-882.

Hanson, A.D., Ritts, B.D., Zinniker, D., Moldowan, J.M., and Biffi, U., 2001, Upper Oligocene lacustrine source rocks and petroleum systems of the northern Qaidam Basin, Northwest China: AAPG Bulletin, v. 85, no. 4, p. 601-619.

Husing, S.K., Hilgen, F.J., Abdul Aziz, H., and Krijgsman, W., 2007, Completing the Neogene geological time scale between 8.5 and 12.5 Ma: Earth and Planetary Science Letters, v. 253, no. 3, p. 340-358.

Jones, C.H., 2002, User-driven integrated software lives: "PaleoMag”: Paleomagnetics analysis on the Macintosh: Computers and Geosciences, v. 28, no. 10, p. 1145-1151.

Kirschvink, J.L., 1980, The least-squares line and plane and the analysis of paleomagnetic data: Geophysical Journal of the Royal Astronomical Society, v. 62, p. 699-718, doi:10.1111/j.1365-246X.1980.tb02601.x.

Kirschvink, J.L., Kopp, R.E., Raub, T.D., Baumgartner, C.T., and Holt, J.W., 2008, Rapid, precise, and high-sensitivity acquisition of paleomagnetic and rock-magnetic data:

Development of a low-noise automatic sample changing system for superconducting rock magnetometers: Geochemistry Geophysics Geosystems, v. 9, p. Q05Y01, doi:10.1029/2007GC001856.

Liu, Z., Wang, Y., Chen, Y., Li, X., and Li, Q., 1998, Magnetostratigraphy and sedimentologically derived geochronology of the Quaternary lacustrine deposits of a 3000 m thick sequence in the central Qaidam Basin, western China: Palaeogeography, Palaeoclimatology, Palaeoecology, v. 140, no. 1-4, p. 459-473.

Lourens, L.J., Hilgen, F.J., Laskar, J., Shackleton, N.J., and Wilson, D., 2004, The Neogene Period, in Gradstein, F.M., Ogg, J.G., and Smith, A., eds., Geological Time Scale: Cambridge, UK, Cambridge University Press, p. 409-440.

Rieser, A., Neubauer, F., Liu, Y., and Ge, X., 2005, Sandstone provenance of north-western sectors of the intracontinental Cenozoic Qaidam Basin, western China: Tectonic vs. climatic control: Sedimentary Geology, v. 177, p. 1-18, doi:10.1016/j.sedgeo.2005.01.012.

Song, T., and Wang, X., 1993, Structural styles and stratigraphic patterns of syndepositional faults in a contractional setting; examples from Qaidam Basin, northwestern China: The American Association of Petroleum Geologists Bulletin, v. 77, no. 1, p. 102-117.

Wang, X., Qiu, Z., Li, Q., Wang, B., Qiu, Z., Downs, W.R., Xie, G., Xie, J., Deng, T., Takeuchi, G.T., Tseng, Z.J., Chang, M., Liu, J., Wang, Y., Biasatti, D., Sun, Z., Fang, X., and Meng, Q., 2007, Vertebrate paleontology, biostratigraphy, geochronology, and paleoenvironment of Qaidam Basin in northern Tibetan Plateau: Palaeogeography, Palaeoclimatology, Palaeoecology, v. 254, no. 3-4, p. 363-385, doi:10.1016/j.palaeo.2007.06.007. 
Wang, Y., Zhen, J., Zhang, W., Li, S., Liu, X., Yang, X., and Liu, Y., 2012, Cenozoic uplift of the Tibetan Plateau: Evidence from the tectonic-sedimentary evolution of the western Qaidam Basin: Geoscience Frontiers, v. 3, no. 2, p. 175-187, doi:10.1016/j.gsf.2011.11.005.

Zhang, W., Appel, E., Fang, X., Song, C., and Cirpka, O., 2012a, Magnetostratigraphy of deep drilling core SG-1 in the western Qaidam Basin (NE Tibetan Plateau) and its tectonic implications: Quaternary Research, v. 78, no. 1, p. 139-148, doi:10.1016/j.yqres.2012.03.011.

Zhu, L., Wang, C., Zheng, H., Xiang, F., Yi, H., and Liu, D., 2006, Tectonic and sedimentary evolution of basins in the northeast of Qinghai-Tibet Plateau and their implication for the northward growth of the plateau: Palaeogeography, Palaeoclimatology, Palaeoecology, v. 241, p. 49-60, doi:10.1016/j.palaeo.2006.06.019.

Table S1. Summary of previous stratigraphic work and age assignments of late Neogene Qaidam Basin strata.

\begin{tabular}{|c|c|c|c|}
\hline Formation & General Description & $\begin{array}{c}\text { Age } \\
\text { Determination } \\
(\mathrm{Ma})\end{array}$ & References \\
\hline \multirow{6}{*}{ Qigequan } & \multirow{6}{*}{$\begin{array}{c}\text { hyper-arid evaporite } \\
\text { (gypsum, halite) and } \\
\text { lacustrine (shale, } \\
\text { mudstone) }\end{array}$} & Quaternary & Song and Wang (1993) \\
\hline & & $1.8-0$ & Reiser et al. (2005) \\
\hline & & $2.5-0$ & Fang et al. (2007) \\
\hline & & $2.6-0$ & Zhu et al. (2006) \\
\hline & & $2.65-0$ & Wang et al. (2012 \\
\hline & & $2.6-0$ & this study \\
\hline \multirow{6}{*}{ Shizigou } & \multirow{6}{*}{$\begin{array}{c}\text { evaporite/freshwater } \\
\text { lacustrine } \\
\text { (mudstone, shale, } \\
\text { marginal lacustrine) }\end{array}$} & $5.3-2.6$ & Wang et al. (2007) \\
\hline & & $5.7-1.8$ & Reiser et al. (2005) \\
\hline & & $5.3-2.45$ & Zhu et al. (2006) \\
\hline & & $8.1-2.5$ & Fang et al. (2007) \\
\hline & & $8.2-2.6$ & Zhuang et al. (2011b) \\
\hline & & $8.2-2.65$ & Wang et al. (2012) \\
\hline \multirow{2}{*}{$\begin{array}{l}\text { Shang } \\
\text { Youshashan }\end{array}$} & \multirow{2}{*}{$\begin{array}{l}\text { freshwater } \\
\text { lacustrine }\end{array}$} & $12.1-5.3$ & $\begin{array}{l}\text { Wang et al. (2007) } \\
\text { Zhu et al. (2006) } \\
\text { Reiser et al. (2005) }\end{array}$ \\
\hline & & 14.9-8.2 & $\begin{array}{l}\text { Hanson et al. (2001) } \\
\text { Wang et al. (2012) }\end{array}$ \\
\hline $\begin{array}{c}\text { Xia } \\
\text { Youshashan }\end{array}$ & lacustrine/fluvial & $\begin{array}{l}\text { early-middle } \\
\text { Miocene }\end{array}$ & \multirow{4}{*}{ see Zhuang et al.(2011b) and references therein } \\
\hline $\begin{array}{c}\text { Shang } \\
\text { Ganchaigou }\end{array}$ & meandering fluvial & $\begin{array}{c}\text { Oligocene- } \\
\text { early Miocene }\end{array}$ & \\
\hline $\begin{array}{c}\text { Xia } \\
\text { Ganchaigou } \\
\end{array}$ & meandering fluvial & $\begin{array}{l}\text { Eocene- } \\
\text { Oligocene } \\
\end{array}$ & \\
\hline Lulehe & braided fluvial & $\begin{array}{l}\text { Early-middle } \\
\text { Eocene }\end{array}$ & \\
\hline
\end{tabular}

Table S2. Summary of magnetostratigraphic data.

\begin{tabular}{|c|c|c|c|c|c|c|c|c|}
\hline Sites & $\begin{array}{c}\text { total } \\
\text { thickness } \\
\text { spanned }\end{array}$ & $\begin{array}{c}\text { sites } \\
\text { used }\end{array}$ & $\begin{array}{c}\text { sites not } \\
\text { used }\end{array}$ & $\begin{array}{c}\text { average site } \\
\text { spacing } \\
\text { used }\end{array}$ & $\begin{array}{c}\text { average } \\
\text { total site } \\
\text { spacing }\end{array}$ & $\begin{array}{c}\text { total } \\
\text { samples } \\
\text { used }\end{array}$ & $\begin{array}{c}\text { total } \\
\text { samples } \\
\text { processed }\end{array}$ & magnetozones \\
\hline 100 & 1672 & 74 & 26 & 22.6 & 16.7 & 107 & 144 & 22 \\
\hline
\end{tabular}


Table S3. Summary of paleocurrent analyses from the Northeast (NE) section. Trough cross-beds provide unidirectional measurements, whereas epsilon cross-beds, channel margins, and ripples provide bi-directional data. Overall directions are in Cartesian coordinates and are based on the combination of uni and bi-directional measurements.

\begin{tabular}{|c|c|c|c|c|c|c|c|}
\hline Location & $\begin{array}{l}\text { strat depth } \\
(\mathrm{m})\end{array}$ & $\begin{array}{c}\text { total } \\
\text { measurements }\end{array}$ & $\begin{array}{c}\text { trough } \\
\text { cross-beds }\end{array}$ & $\begin{array}{c}\text { epsilon } \\
\text { cross-beds }\end{array}$ & $\begin{array}{l}\text { channel } \\
\text { margins }\end{array}$ & ripples & $\begin{array}{c}\text { Overall } \\
\text { Direction }\end{array}$ \\
\hline Unit 3 & $1500-1725$ & 14 & 8 & 0 & 0 & 6 & SSW \\
\hline Unit 3 & $1235-1500$ & 37 & 18 & 17 & 2 & 0 & $\mathrm{~S}$ \\
\hline Unit 2 & $920-1235$ & 8 & 8 & 0 & 0 & 0 & WSW \\
\hline Unit 1 & $760-920$ & 0 & 0 & 0 & 0 & 0 & na \\
\hline Unit 2 & $495-760$ & 47 & 34 & 13 & 0 & 0 & SW \\
\hline Unit 1 & $175-495$ & 18 & 14 & 2 & 2 & 0 & WSW \\
\hline Unit 1 & $0-175$ & 9 & 7 & 2 & 0 & 0 & $E$ \\
\hline
\end{tabular}

Table S4. Carbonate phases observed in the carbon and oxygen istope samples.

\begin{tabular}{|c|c|c|c|c|c|c|c|}
\hline OurLabID & Sample ID & Phase & alteration & OurLabID & Sample ID & Phase & alteration \\
\hline C-3819 & NE01 & cement & & C-3852 & NE45 & cement & \\
\hline C-3820 & NE02 & cement & & C-3853 & NE47 & cement & \\
\hline C-3821 & NE03 & cement & & C-3854 & NE54 & cement & \\
\hline C-3822 & NE04 & cement & & C-3855 & NE59 & micrite & \\
\hline C-3823 & NE05 & cement & & C-3856 & NE62 & micrite & \\
\hline C-3824 & NE06 & cement & & C-3857 & NE65 & micrite & \\
\hline C-3825 & NE07 & cement & & C-3858 & NE67 & cement & \\
\hline C-3826 & NE08 & cement & & C-3859 & NE69 & micrite & \\
\hline C-3827 & NE09 & cement & & C-3860 & NE71 & micrite & \\
\hline C-3828 & NE10 & cement & & C-3861 & NE72 & cement & \\
\hline C-3829 & NE11 & cement & & C-3862 & NE73 & micrite & \\
\hline C-3830 & NE12 & cement & & C-3863 & NE75 & cement & \\
\hline C-3831 & NE13 & cement & & C-3864 & NE78 & cement & \\
\hline C-3833 & NE14 & cement & & C-3865 & NE81 & cement & \\
\hline C-3834 & NE15 & cement & & C-3866 & NE82 & cement & \\
\hline C-3879 & NE16 & cement & & C-3867 & NE83 & micrite & \\
\hline C-3835 & NE17 & cement & & C-3868 & NE84 & cement & \\
\hline C-3836 & NE18 & cement & & C-3869 & NE85 & cement & \\
\hline C-3838 & NE20 & cement & & C-3870 & NE86 & micrite & \\
\hline C-3839 & NE21 & cement & & C-3871 & NE88 & cement & \\
\hline C-3840 & NE22 & cement & & C-3872 & NE89 & cement & \\
\hline C-3841 & NE25 & cement & & C-3880 & NE92 & cement & \\
\hline C-3842 & NE26 & micrite & dolomite alteration & C-3873 & NE93 & cement & \\
\hline C-3843 & NE27 & cement & & C-3874 & NE94 & micrite & hematite/calcite \\
\hline C-3844 & NE29 & cement & & C-3875 & NE95 & micrite & \\
\hline C-3845 & NE31 & cement & & C-3876 & NE98 & micrite & \\
\hline C-3847 & NE34 & cement & & C-3877 & NE99 & micrite & \\
\hline C-3848 & NE39 & cement & & C-3878 & NE100 & cement & \\
\hline C-3851 & NE43 & cement & & & & & \\
\hline
\end{tabular}

